\title{
Systems, Networks, and Policy
}

\author{
Jeffrey Johnson, Joyce Fortune, and Jane Bromley
}

\begin{abstract}
Systems theory is fundamental to understanding the dynamics of the complex social systems of concern to policy makers. A system is defined as: (1) an assembly of components, connected together in an organised way; (2) the components are affected by being in the system and the behaviour of the systems is changed if they leave it; (3) the organised assembly of components does something; and (4) the assembly has been identified as being of particular interest. Feedback is central to system behaviour at all levels, and can be responsible for systems behaving in complex and unpredictable ways. Systems can be represented by networks and there is a growing literature that shows how the behaviour of individuals is highly dependent on their social networks. This includes copying or following the advice of others when making decisions. Network theory gives insights into social phenomena such as the spread of information and the way people form social groups which then constrain their behaviour. It is emerging as a powerful way of examining the dynamics of social systems. Most systems relevant to policy have many levels, from the individual to local and national and international organisations and institutions. In many social systems the micro, meso and macrolevel dynamics are coupled, meaning that they cannot be studied or modified in isolation. Systems and network science allow computer simulations to be used to investigate possible system behaviour. This science can be made available to policy makers through policy informatics which involves computer-based simulation, data, visualisation, and interactive interfaces. The future of science-based policy making is seen to be through Global Systems Science which combines complex systems science and policy informatics to inform policy makers and facilitate citizen engagement. In this context, systems theory and network science are fundamental for modelling far-from-equilibrium systems for policy purposes.
\end{abstract}

\footnotetext{
J. Johnson $(\varangle) \bullet$ J. Fortune $\bullet$ J. Bromley

Faculty of Science, Technology, Engineering and Mathematics, The Open University, Milton Keynes, UK

e-mail: jeff.johnson@open.ac.uk; joyce.fortune@open.ac.uk; jane.m.bromley@open.ac.uk
}

J. Johnson et al. (eds.), Non-Equilibrium Social Science and Policy, Understanding Complex Systems, DOI 10.1007/978-3-319-42424-8_8 


\section{Introduction}

A major challenge for policy makers is the confidence they can have that a policy will deliver the intended outcomes and not generate unintended consequences. For millennia the projected outcome of policy has been based on conviction, rhetoric, evidence, logical analysis and calculation. Modern information and communication technologies provide powerful support for the last three of these, based on the organising principle of 'system'.

A system is a collection of interacting elements, with them all affecting and being affected by the behaviour of the whole. Systems may have intermediate levels of organisation as subsystems. People are the key elements of social systems, but they may also include physical objects such as buildings, machines, and infrastructure such as airports; biological objects such as plants and animals; and natural subsystems such as the land, oceans, and the atmosphere. Together these components often form complex multilevel systems.

The definition of system in this chapter includes knowing why the system is of particular interest. In policy-driven science this is an essential guiding principle for deciding which elements and relationships are relevant.

Over the last quarter century the notion of interaction in systems has become much better understood through the development of network science. Networks have generic properties that can make the behaviour of systems more understandable. These include many structures and properties based on concepts of connectivity, and ways of representing communities and their interactions.

Social systems have such variety and combinatorial complexity that their trajectory through time may never be repeated. Although some patterns may be discerned, precise outcomes are unpredictable.

Unpredictability is a fundamental problem for policy-how can one choose between policies when their outcomes are uncertain? Systems and network theory attempt to answer this question. Systems diagrams can give insights into the dynamics of systems including making more apparent their interacting feedback loops. Since these can become very complicated computer programs are often needed to investigate their behaviour and examine possible system futures by iterated computation.

When systems behave 'normally' their variables may stay within narrowly defined bounds and be close to equilibrium. The policy challenge for science is not to say what will happen when a system is behaving 'normally' or close to a predictable equilibrium, but to say when a system will behave 'abnormally' and change its behaviour to another equilibrium or even non-equilibrium state.

Systems and complex network theory give policy makers a way of investigating the possible outcome of their policies. They can inform policy makers when social systems are close to equilibrium but are more concerned with the far from equilibrium dynamics of local and global change and extreme events Evidencebased policy aims to use the best scientific knowledge with the best available data. This requires interdisciplinary complex systems science which aims to integrate 
knowledge from all domains in support of policy. This chapter shows how systems and network science can contribute to this.

\section{Policy}

Policy involves making a decision to change a system, and planning and managing the execution of that decision. In the words of Herbert Simon, it is taking action to make the system as it ought to be [31]. How a system 'ought' to be depends on the interests of its stakeholders. In democracies the conflicting interests of stakeholders are resolved through constitutions, custom and elections. Here the focus is on modelling social systems in order to best inform policy making.

The outcome of a policy is usually monitored. If a system is not evolving as anticipated new interventions may be made to 'put it back on track'. 'Take action, monitor outcomes, respond with new actions, monitor outcomes,...' is an example of a feedback cycle as explained in the next section.

Policy requires a view of systems that overcomes the academic divisions between social, economic, political, biological, physical, medical, and engineering systems. For example, a policy decision to build a new hospital could involve all of these and others too, such as the legal, planning and transportation systems. How can systems with such heterogeneous parts be represented in the policy process? In particular:

How can policymakers be confident that a policy will deliver the intended outcomes, and not generate unintended consequences?

Systems and network theory aim to provide methods to help answer this question.

\section{Systems}

Of many more or less equivalent definitions of systems, Bignell and Fortune [8] define a system as

1. an assembly of components, connected together in an organised way;

2. the components are affected by being in the system and the behaviour of the systems is changed if they leave it;

3 . the organised assembly of components does something; and

4. the assembly has been identified as being of particular interest.

These characteristics are particularly applications-oriented where policy considerations determine what is of particular interest and what the system does that is of particular interest. This guides the identification of relevant system components by asking does a candidate component affect what the system does and how is that component affected by being part of the system? Then there is the question as to how the components are connected-how they are related to each other, and how 
they are affected by being part of the system. This leads to the question of how the organised assembly does those things that are of particular interest. In other words how does the system behave? If this is known there is the possibility of taking action to make it behave in specific 'desirable' ways.

\subsection{System Diagrams}

Systems diagrams have evolved as a way of providing a pictorial overview of systems and their dynamics. Typically the relationships between the elements of the system are drawn as arrows between them. The process of drawing systems in this way can be very illuminating to the analysts and provides a way of communicating to others the insights gained.

\subsubsection{The Bovine Tuberculosis-Badger Culling System}

The 'Bovine Tuberculosis (BTB)_Badger-Culling' system has been identified of particular interest by UK farmers who want to eradicate wild badgers because they can infect their cows, thus causing their herds to be slaughtered. The system components include badgers, cows and bovine tuberculosis and they are connected by the propositions that badgers infect cows and that removing badgers from the system would change its behaviour by removing bovine tuberculosis. Badgers are a protected species and to kill badgers legally it is necessary to have a licence, so the farmers need culling badgers to become government policy.

To test this policy option the then Labour Government commissioned a Randomised Badger Culling Trial 1998-2007 [9]. This study concluded that badgers do contribute to the spread of bovine tuberculosis but culling is unlikely to make a significant difference, and better animal management practice was recommended to impede the spread of the disease. Badgers are territorial animals and killing less than $70 \%$ disrupts their social structures with survivors roaming further afield, exacerbating the problem. On this evidence the then Labour government decided not to proceed with a cull.

Following the 2010 election of a Conservative-led coalition more sympathetic to the farming lobby, in 2011 it was decided to allow badger culls [11]. However, any official badger cull had to expect violent animal rights protests requiring a major commitment of police and security resources, thus extending the policy-relevant components and relationships in this system, and increasing its complexity.

In 2012 the programme of badger culling had to be abandoned. As the UnderSecretary of State explained: "The exceptionally bad weather this summer has put a number of pressures on our farmers and caused significant problems. Protracted legal proceedings and the request of the police to delay the start until after the Olympics and Paralympics have meant that we have moved beyond the optimal time for delivering an effective cull. We should have begun in the summer." [16] 
Thus the policy-relevant components in the BTB-Badger Culling system suddenly included the Olympic Games and the weather! This illustrates the 'messy' reality of the systems policy makers need to consider. Here the objective scientific evidence (biological) was just part of a wider social system that behaved in unexpected ways. A better understanding of the interacting social systems could have spared the policy makers an expensive and embarrassing reversal that pleased neither friends nor opponents.

Systems theory makes extensive use of diagrams such as that in Fig. 1. The words, often enclosed in boxes, represent the components of the system and the arrows the interactions between the components. To the right of the dashed line is the relationship that drives the whole system: badgers cause BTB. This results in sufficient farmers losing their herds for their representatives to put intense pressure on the Government for a badger cull. The farmers argue that a badger cull will reduce a badger population, thus removing a cause of BTB. The Government responds to the pressure by authorising a badger cull and paying farmers to implement the cull. However there is also the relationship between the animal rights protesters who will disrupt badger culls, and the police and security services who are expected to contain and control this disruption so that the cull can go ahead. All this was the basis for the 2011 policy to go ahead with the cull.

To the left of the dashed line is the July-August 2012 London Olympic games which is an important part of this system. It had been known for a long time that security considerations would stretch the police and security services to the limit, but the 2011 policy document [11] made no mention of this. Also not mentioned in the policy document was the prospect of bad weather disrupting the proposed cull, again to the left of the dashed line in Fig. 1.

When these two components are included in the system it is clear that the badger cull cannot begin before late August 2012 because the police and security services

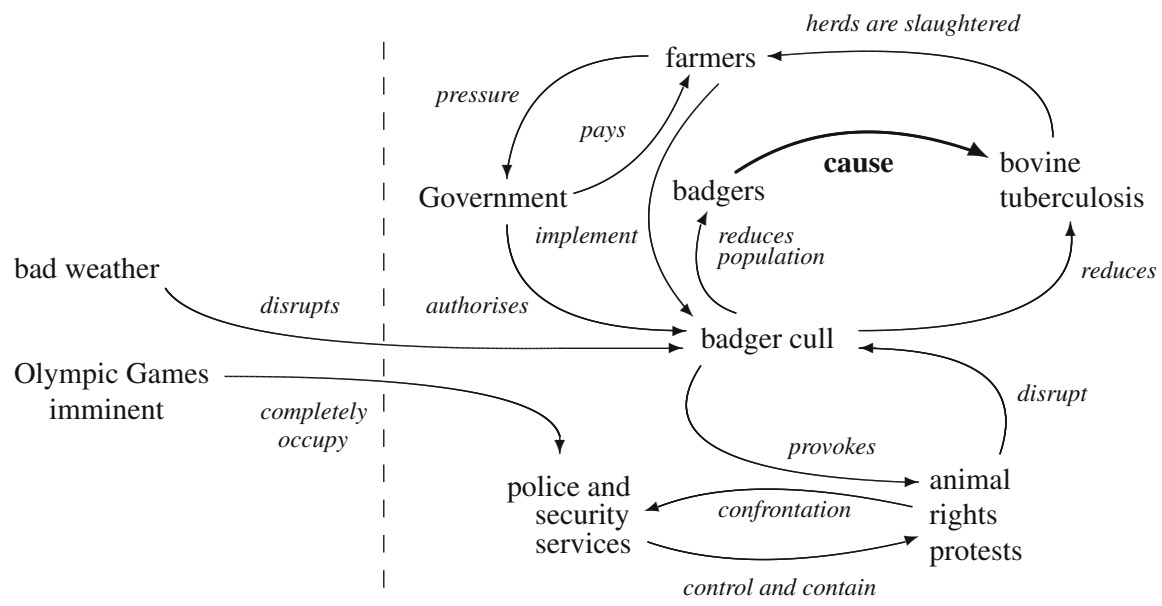

Fig. 1 A system diagram for the badger-cull policy 
would be pre-occupied with Olympic games but the bad weather meant that the farmers and others licensed to execute the cull were not in a position to do so. Hence the cancellation of the cull announced in October 2012 [16].

\subsection{Feedback}

\subsubsection{Negative Feedback}

Negative feedback, as illustrated in Fig. 2, is well-known to policy makers and those who execute policy. It is how they 'steer' the system and keep it 'on course' towards the desirable outcomes. It is analogous to driving a car when, for example, monitoring performance shows it is going too far to the left, requiring the corrective action of steering to the right until it is going in the desired direction. 'Negative' means that the corrective action is in the 'opposite direction' from any observed error.

In social systems interaction takes time, and network dynamics often include delays. For example, after making a policy intervention, it takes time before monitoring will show any effects. It will take time to determine any deviations from what is desired and to plan new policy actions. After that it will take time to implement the plan. Delays can be fundamental to a system's dynamics, and they are represented by two short parallel lines on the interaction arrows.

\subsubsection{Positive Feedback}

Positive feedback reflects escalating change, e.g. a run on a bank. Figure 3 shows concern that the bank will crash causing people to queue to withdraw their money. The sight of a queue outside the bank causes even more people to join it, causing greater concern that the bank will crash, causing even longer queues until eventually the bank does indeed fail. The ' + ' signs near the arrow ends signify increasing effects. The ' $\mathrm{R}$ ' in a circular arrow signifies a reinforcing feedback loop, spiralling with ever increasing effects. Positive feedback cannot go on forever and eventually the system changes, sometimes catastrophically such as it crashing or flying apart.

Fig. 2 Negative feedback is usually used to keep systems close to a desired state

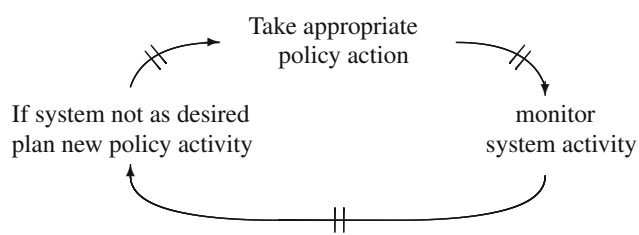




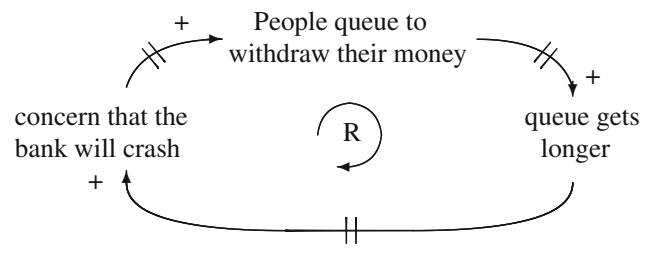

Fig. 3 Positive feedback during a run on the bank

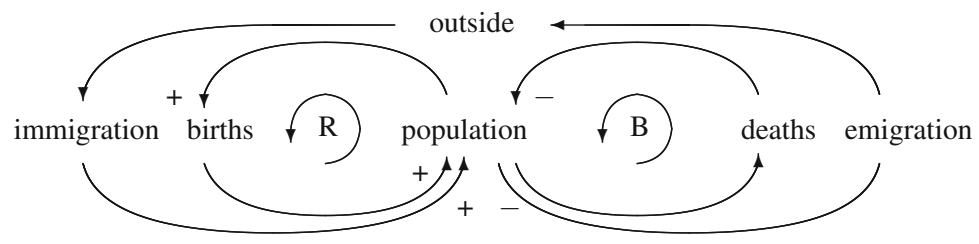

Fig. 4 Interacting feedback loops

\subsubsection{Interacting Feedback Loops}

Real systems normally have combinations of positive, negative and mixed feedback loops. For example, Fig. 4 shows the reinforcing feedback loop of births interacting with the balancing feedback loop of deaths, signified by the letter ' $\mathrm{B}$ ' inside a circular arrow. If the numbers of births and deaths were perfectly balanced the population would be in equilibrium, but this is rarely the case. The population also depends on immigration and emigration. This system has a number of interacting feedback loops, and this can make short and long-term forecasts of future population difficult.

\subsubsection{Oscillating Feedback Mechanisms}

Figure 5 shows two interacting positive feedback mechanisms. On the left is a bull market with people believing that prices will increase and buying, the increase demand causing prices to increase. On the right is a bear market with people believing that prices will fall and selling, causing a surplus supply and falling prices.

In practice these two subsystems tend to oscillate as 'market sentiment' transforms the belief that prices will increase to the belief that prices will fall, and vice versa. The dynamics of these changes are partly due to the narratives circulating as the system evolves, as discussed in the chapters by Ormerod and Nowak in this volume [27, 29]. 

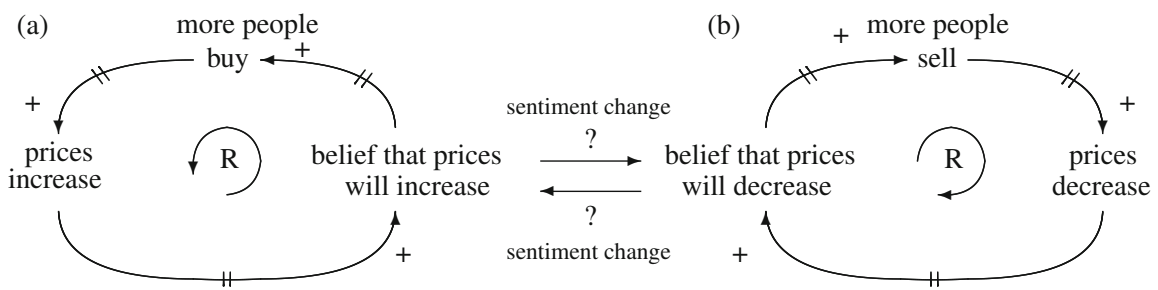

Fig. 5 Oscillation between two positive feedback loops linked by the dynamics of sentiment change. (a) Bull market. (b) Bear market

\subsection{Computing System Behaviour}

Feedback is a major cause of systems complexity. When the behaviour of systems emerges from many interactions with many coupled feedback loops, there are no formulae that can predict it. Computer simulation by iterative computation based on data has been developed in complex systems science as a systematic way to investigate how heterogeneous multiple feedback interaction systems might behave.

\subsubsection{Coupled Populations}

Figure 6a uses data from the Hudson Bay Company to show predator-prey interaction between the populations of snowshoe hares and Canadian lynx. When the number of hares increases the number of lynxes increases, but more lynxes means that more hares are eaten reducing the population of hares. Figure $6 \mathrm{~b}$ shows the observed populations of hares and lynx from 1845 to 1925 . This shows that, far from there being an equilibrium, the coupled populations fluctuate considerably due to the time lags of the population interactions. Other predator-prey systems have been studied extensively by computer simulation and they show the same oscillations in populations as those in Fig. 6.

\subsubsection{Computing State Transitions}

If the observed state of a system at various points in time is recorded in some way the resulting data almost always contain measurement errors, and the observed state is always an approximation to the 'real' state.

Computer simulation involves modelling systems in discrete time. It is assumed that a clock is ticking and that, given the knowledge of the state of the system now, it is possible to compute the state of the system at the next tick of the clock. This assumes each element of the system has a state recorded in some way, and that the transition from one state to a state one tick ahead can be computed for the next tick of the clock. This computation depends on the current state of the particular element 

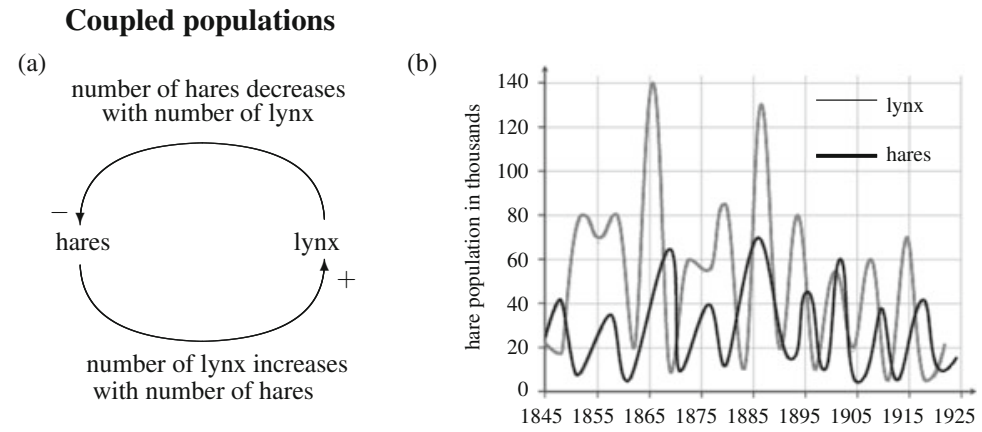

Fig. 6 The co-evolution of coupled populations. (a) Coupled feedback loop. (b) Coupled populations of snowshoe hares and Canadian lynx

and all the states of all the elements it interacts with. Then the state of the particular element can be updated at each tick of the clock.

After the first computation, another will give the state of the system after two ticks of the clock. In general many iterations of the computation will give the state of the system many ticks later. For iterated computations the assumed state of the system at the beginning is called the initial state or the initial conditions of the system.

The state calculated for any particular time in the future could be considered a point prediction of the state of the system at that point in time. A single point prediction generated by iterated computation may be very different from the observed state of the real system, and by itself of little use in policy making.

\subsubsection{Boundedness and Phase Change}

The variables representing a system are said to be bounded if they always stay within a finite range. In practice this means that they do not diverge to infinity and get so large that the system flies apart.

For example, the populations in predator-prey systems may become very large but system properties ensure they will reduce in the future. The maximum level of the population of prey is bounded above by the rate of predation. Similarly the maximum level of the population of predators is bounded above by the number of available prey. The minimum populations of both predators and prey are bounded below at two animals, one male and one female, to breed.

Sometimes in simulations the populations of both prey and predator die out. This is an extreme example of a phase change [3], where the system ceases to exist. Other example of phase changes in social systems include: the transition from high speed low density traffic to a low speed high density traffic jam; divorce, riots; bankruptcy; change of government; and disruptive technological innovation. 


\subsubsection{Sensitivity to Initial Conditions}

Sensitivity to initial conditions means that a small change in initial conditions can result in a big change in system behaviour. For example, if you had left home $5 \mathrm{~min}$ earlier you would have caught the flight and not been a day late. A system is said to be sensitive to initial conditions if a small difference in the measurement of its state now causes a big difference in the point-prediction of its state at some future time.

A continuous system that is bounded and sensitive to initial conditions is said to be chaotic. This means that after some time-horizon its state will be unpredictable, e.g. after some period of time the precise rain-or-shine weather on a given day in many parts of the world cannot be predicted, although the likelihood of particular weather states can be usefully estimated.

For discrete systems that are sensitive to initial conditions, a single point prediction based on iterated computation has almost no value after some timehorizon. However, many point predictions may give an overview of the 'space' of possible futures at future times, and this can be useful to policy makers. For example:

1. if the desired outcome of a policy is never generated by iterated computation this is strong evidence for a policy maker that the action proposed may not work

2. iterated computation may generate outcomes that had not been envisaged and give insights into 'unknown unknowns'

3. iterated computation may show that the space of possible futures for a policy contains some very bad or dangerous outcomes, and taking a bet on that policy would be irresponsibly risky

4. iterated computation may show that a policy is indeed highly likely to achieve its objectives.

Sensitivity to initial conditions thus means that systems have to be simulated across a wide range of initial conditions to give an insight into the space of possible futures. Sometimes these are very different, and there may be no way of knowing which is most likely to occur. However, it can be very useful to discover that systems have behaviours not previously imagined and simulation can give insights into them.

\subsubsection{System Dynamics}

The theory of system dynamics developed by Forrester [14] quantifies the states of the elements and the interaction transitions between them. The underlying idea is that components of the system have properties represented by numbers, that the state of the system at any time can be represented by those numbers, and the interactions between the components can be represented by mathematical functions that map the state at one tick of the clock to state at the next. Figure 7 shows two computer simulations based on a systems model and the iterated computation [14].

Forester's systems dynamics was the basis of the study by the Club of Rome resulting in the publication by Meadows et al. of the controversial report The Limits 

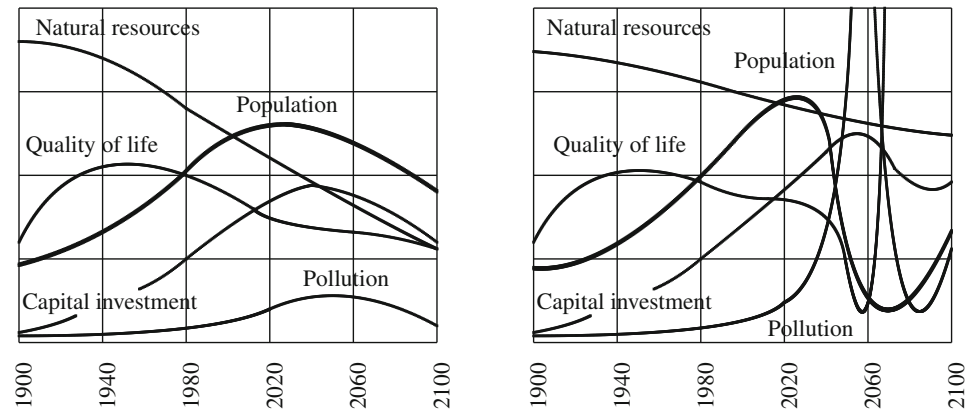

Fig. 7 Part of Forrester's 1971[14] simulations of world dynamics

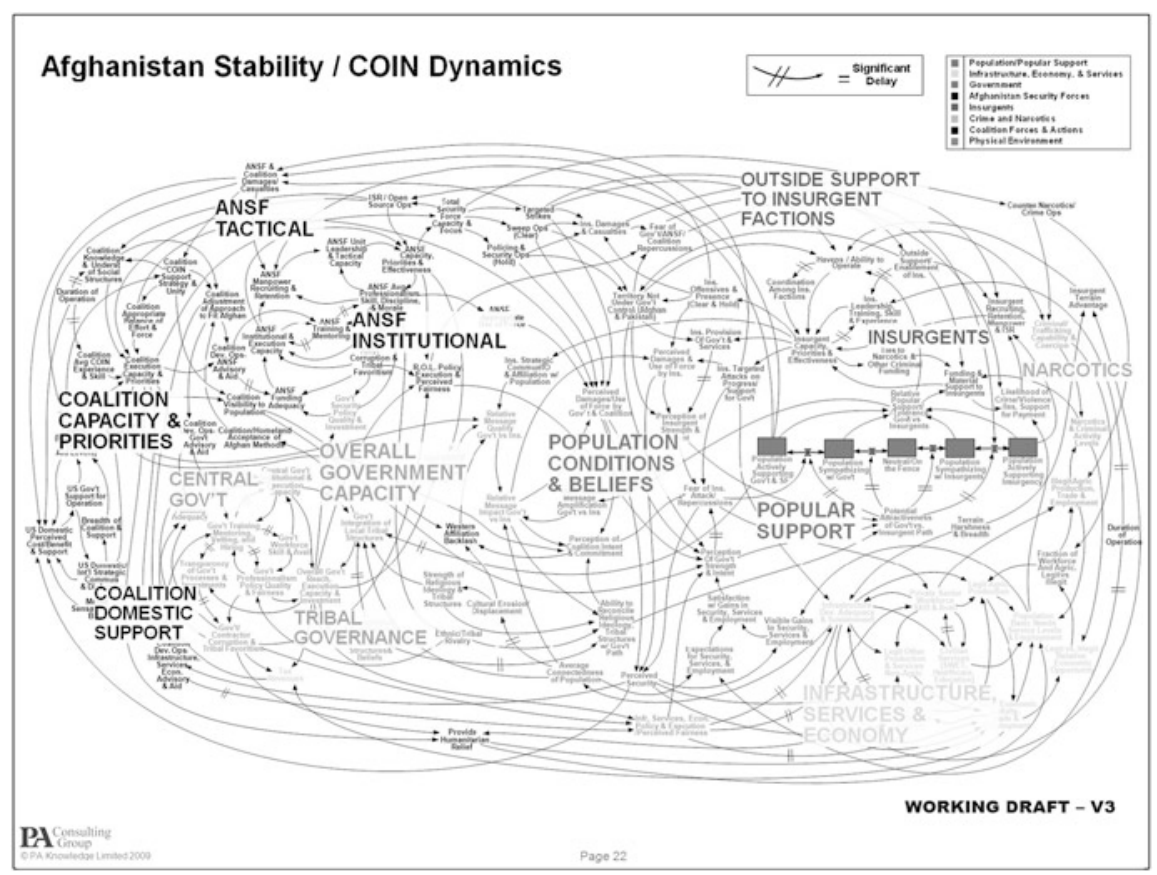

Fig. 8 Complicated systems diagrams can be hard to understand

to Growth [22, 23]. This study has been widely criticised from technical and policy perspectives but most critics miss the point that the purpose of this early study was to use systems dynamics to explore possible futures rather than to predict any particularly likely future.

In another application of systems dynamics, when US Gen. McChrystal, the US leader in Afghanistan, was shown the diagram portraying American military strategy in Fig. 8 The New York Times wrote that it looked more like a bowl of spaghetti and that General McChrystal had remarked 'When we understand that slide we'll have won the war'. 
In a robust defence of the diagram, published in the Times Newspaper [26], Jon Moynihan of PA Consulting Group wrote

You printed what has been dubbed on the internet a 'spaghetti' chart, depicting the current Afghan environment ? However, ours was far from being over-simplistic PowerPoint, using instead a well-known technique - system dynamics - to review a highly complex situation. Unlike linear thinking, the default mode of the human brain, system dynamics thinks about repercussions and occasionally unintended consequences of actions. ... do we really want simplistic philosophies to win out ... Do we want strategies developed that take no account of complexity and the sometimes counterintuitive outcomes of well-intentioned actions?

The 'spaghetti' criticism is easy to make, but assuming systems diagrams contain relevant components and relevant relationships, whether or not a systems approach is taken, this information is necessary if not sufficient to understand systems and their dynamics.

\subsection{Equilibrium}

The idea of equilibrium can be illustrated by a displacing a ball bearing in a bowl as shown in Fig. 9a where gravity restores it to an equilibrium at the bottom of the bowl. Here gravity acts as a negative feedback mechanism that forces the ball back to its equilibrium state. Figure $9 \mathrm{~b}$ shows a ball balanced on a point. This a dynamic equilibrium. Any slight deviation from the equilibrium will result in the ball falling and equilibrium being lost. Here gravity induces positive feedback.

A problem with equilibrium theory for a social system is that it would never change. This is taken into account by the theory of punctuated equilibrium, which allows that systems may remain in more or less the same state for long periods of time and then change to a new equilibrium as shown in Fig. 9c. For example, a price may be stable for a long period of time and then can change considerably with the advent of a new technology.

In equilibrium systems it is not necessary that what is being measured is constant. It is sufficient that it remain close to the equilibrium state. For example, the price of petrol in a city may vary from place to place, but market forces will keep it in

(a)

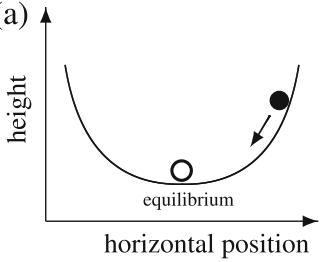

(b)

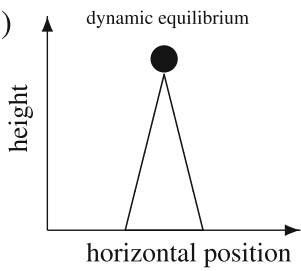

(c)

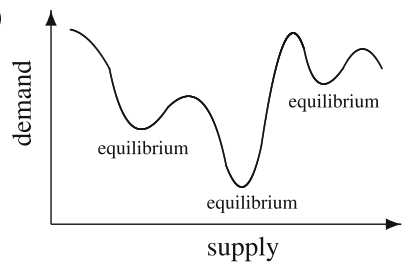

Fig. 9 Equilibrium between variables in systems. (a) Ball in bowl. (b) Balanced ball. (c) Punctuated equilibrium 

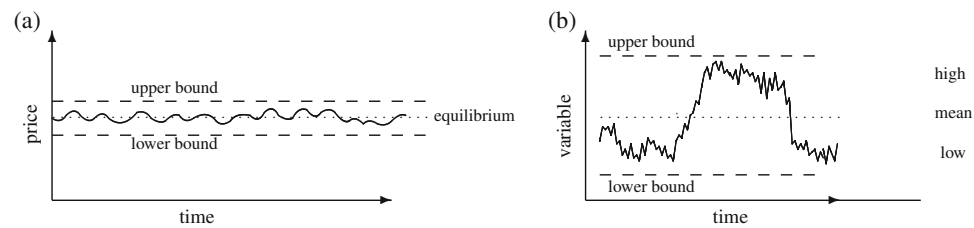

Fig. 10 Bounded systems and equilibria. (a) System within bounds close to equilibrium. (b) Multiphase bounded system

bounds close to an equilibrium price Fig. 10a. One outlet is unlikely to be able to charge twice the price as another for any length of time.

Figure 10b shows another bounded system variable. Here there appear to be two equilibrium states, one high and the other low, on either side of the mean. From a policy perspective the important question is if and when the system will move from one 'equilibrium' phase to another.

\subsection{Multilevel Systems}

The UK currently faces a crisis in its hospitals due to 'bed blocking' by elderly patients whose clinical treatment is complete but who cannot manage by themselves at home and for whom there is no place in the non-clinical welfare system [17].

Statistics from the Department of Health show that in a year 680,000 elderly people treated by the National Health Services (NHS) can be detained on wards for weeks, even though they are well enough to be looked after in a care home or at home with social services support. It is reported that "the loss of almost 50,000 council and private care home beds over the last 5 years, rising demand and social services budget cuts are blamed for the shambles. Meanwhile, cancelled operations-directly linked to a lack of beds-have soared from 3733 during the first quarter of 2000-to 4881 today (2015)." [21]. The opposition Labour Party blames the delays and bed-blocking on cuts of $£ 3.5$ billion to social care budgets since 2010 that have caused more elderly people to be admitted to hospital instead of being cared for at home. [25]

The problem is that the NHS pays when patients are in hospital, but when they are in community care the local council pays. The NHS and councils have different staff, separate budgets, and different priorities. Also, when a patient is transferred to a hospital in another county, another NHS trust must pay for them. To further complicate matters, if an elderly patient from one town is admitted to hospital in another they cannot access community care services there because they are not a resident. Instead, they must wait for someone from their local authority to come to assess them and organise care in their own community. This process can take a long time and it means patients can be stuck blocking a hospital bed for weeks. 
This problem illustrates the nature of multilevel systems in policy. On the one hand the government funds directly and is responsible for the NHS, while on the other local authorities provide social care alongside many other services. Here the NHS subsystem and the Local Government subsystem have different funding structure, different cultures and different incentives. In particular the Local Authorities have been subjected to major cuts in government grants under the austerity policy introduced in 2010 , and they make these cuts according to local priorities.

The 'Better Care Fund: Policy Framework' [5, 15] gives details of the plan to make Department of Health and the Department for Communities and Local Government work together to improve care in general and solve the bed-blocking problem in particular. The stakes are high-if the current problems with bed blocking are not resolved it could be a determining factor at the next election. An important question for the UK Government is whether or not this policy will work?

The analysis of this system in the policy document is mostly verbal, including discussion of new budget allocations. There is no discussion of the micro or mesolevel dynamics of hospital and local councils working together. In particular there is no requirement for the welfare subsystem to provide sufficient beds to allow hospitals to discharge all patients from clinical beds into the community. With the information available one cannot know how this complex heterogeneous multilevel system will behave.

\section{Networks}

According to the definition in Sect.1, every system can be represented by a connected network. The elements of the system form the vertices of the network, usually represented by a small circle referred to as a node. When two elements interact a line, referred to as an edge or a link is drawn between them. Figure 11a shows an ego network for the centre node, similar to a person surrounded by their friends. Figure $11 \mathrm{~b}$ shows a clique with all the vertices connected, as with a tight social structure of friends or associates. There are six vertices so this is a 6-clique. Weaker kinds of clique are also possible such as groups of people connected to allbut-one of each other. Figure 11c shows a network in which every node is connected to every other node. Two elements are connected if there is a path between them. The distance between two nodes is the number of links in the shortest path between them, e.g. the distance between $A$ and $B$ is 14 links. The diameter of a network is the longest distance between all pairs of its nodes. In this network the longest shortest paths have length 14, so the network has diameter 14 . 

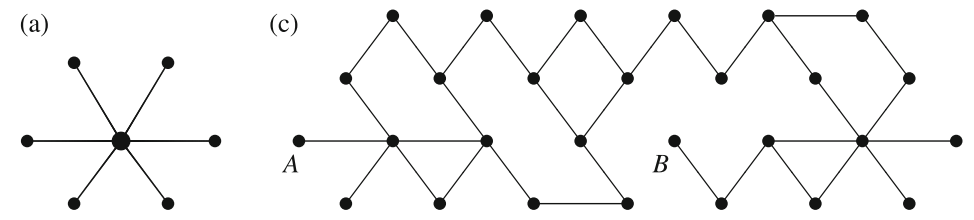

(b)

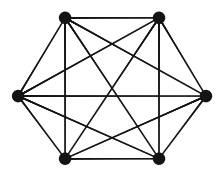

(d)

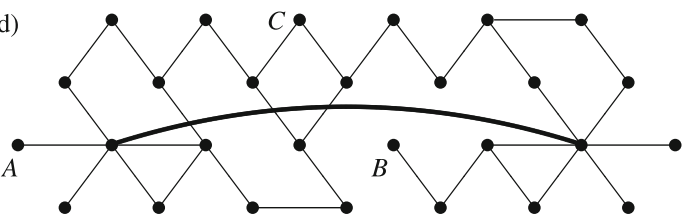

Fig. 11 Links may be curved, and long links reduce path lengths and the diameter of networks. (a) An ego network. (b) A clique. (c) A network with diameter 14, the length of a path from $A$ to B. (d) A long link in a small world network

\subsection{Small World Networks}

The degree of separation between two people is the number of steps or links that connect them. Remarkably, for most pairs of people in the world this is about six. This is called the small world property. It occurs when vertices for local community structures are connected by short paths, and there exist a few long links connecting distant communities. For example, Fig. 11d shows a long link as a curved line. It reduces the diameter of the network from 14 in Fig. 11c to 9 in Fig. 11d, the longest path now being between $B$ and $C$. In social systems long links would correspond to a friend having an aunt in Australia, or a colleague in Washington or a schoolfriend living Thailand.

\subsection{Statistical Properties of Networks}

The number of links attached to a vertex is called its degree. For example, the central vertex in the ego network in Fig. 11a has degree 6. In many social networks the distribution of degrees has a long tailed distributions as illustrated in Fig. 12b. This is because in most social structures a few people have many connections and many people have a few connections.

Traditionally social science focussed on statistical properties of populations which have well defined peaks such as the bell shaped normal curve and its skewed variations, e.g. the height of the population, age at first marriage, and number of attempts at the driving test. Complex systems science has shown that many distributions do not following this pattern but have long tails, or are scale free. E.g., a person new to an area might try to meet a person who already knows a 
(a)

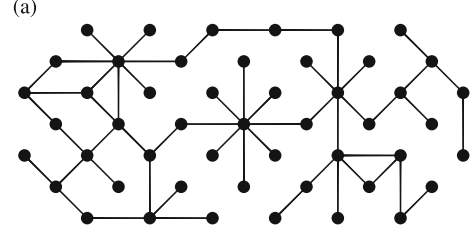

(b)

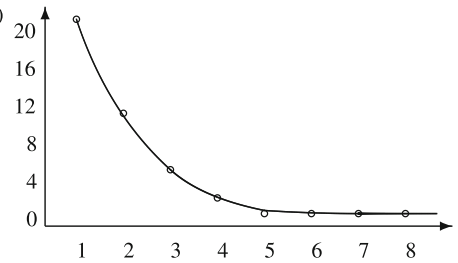

Fig. 12 Many social networks have long tailed distributions of their vertex degrees. (a) A network. (b) Long tailed distribution of degrees

lot of other people, thereby increasing further the number of people that gregarious person knows.

Barabasi proposed preferential attachment as a possible mechanisms underlying the long tailed distributions [4]. For example, an airline in a small town is likely to prefer having a new route to a large city airport rather than another small town airport. In general, when new links are made in the system, they attach to vertices which already have high degree. An equivalent of this is the rich getting richer!

\subsection{Orientation and Relational Asymmetry}

Often the order of edge vertices matters, e.g. $\langle a, b\rangle$ represents $a$ lending money to $b$, this is different from $b$ lending money to $a$. When $\langle a, b\rangle \neq\langle b, a$,$\rangle the edges are said$ to be oriented. Often this is represented in network diagrams by arrows for edges.

\subsection{Percolation}

Information transmission can cascade through networks as one person interacts with others. Social networks support other kinds of transmission, including the spreading of diseases, gossip, and opinions. Things pass from one person to another according to their network structure, e.g. good jokes rapidly cascade through a population. In some cases you do not even need to know the other person, e.g. to catch flu you only need to be linked by being in the same place at the same time. Transmission of anything through networks from one vertex to another is known as percolation.

\subsection{Motifs}

Network motifs are meaningful substructures that appear in networks, e.g. cliques. Figure 13a shows an old fashioned 'Male-Female three couple' dinner party motif. Figure 13b shows a 'gang of boys with leader' motif. Removing the leader destroys 

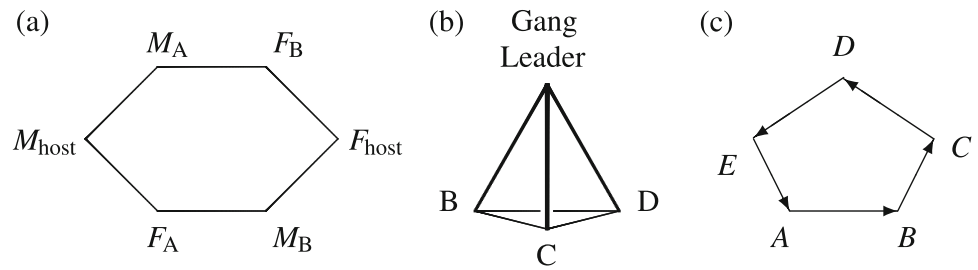

Fig. 13 Motifs as meaningful substructures appearing in networks. (a) dinner party motif. (b) Gang motif. (c) Remuneration motif

this stable structure unleashes unpredictable succession dynamics as the remaining substructures reconfigures to a similar leader-led motif [18]. Figure 13c shows an 'old boys network' motif in which company directors discretely sit on each other's remuneration committees and award each other high remuneration packages.

\subsection{Multiplex Networks}

Simple conventional networks are defined by single relations such as two people being friends but often there are many interacting relations determining the behaviour of systems. For example a 'family system' relation can interact with an 'employment structure' relation to create the phenomenon of 'nepotism'. When modelling social systems it is becoming increasingly important to take many relations into account. For example, when forecasting road traffic, relations such as those between people and their jobs, children and their schools, shoppers and shops combine, as illustrated by a parent driving their partner to work, dropping of the children at school, calling in at the supermarket and ending their trip at the golf course. Networks that include many relations are multiplex networks [20].

\subsection{Hypergraphs and Simplicial Complexes}

To date network theory has focussed on relations between pairs of entities. Certainly many social relations are binary and involve just two entities. However there are many examples of interactions between more than two entities.

Hypergraphs [7] were an early attempt to represent relations between many things, e.g. $\{a, b, c$,$\} could be a hypergraph edge representing the 3$-ary relation between $a, b$, and $c$. e.g. ' $a$ is the team leader of $b$ and $c$ '. However, this is not that same as ' $c$ is the team leader of $a$ and $b$ '. A hypergraph edge, $\{a, b, c\}$ where the order of the vertices matters is called a simplex and represented as $\langle a, b, c\rangle$. In general $\langle\ldots, a, \ldots, b, \ldots\rangle \neq\langle\ldots, b, \ldots, a, \ldots\rangle$. Simplices are oriented and 
generalise oriented 1-dimensional edges $\langle a, b\rangle$, e.g. $\langle a, b, c\rangle$ is a 2-dimensional 'filled in' triangle, and $\langle a, b, c, d\rangle$ is a solid 3-dimensional tetrahedron.

A set of objects is related under a higher dimensional relation if removing any of them causes the relation to break down, e.g. remove the piano, viola, violin or cello from a piano quartet and it ceases to be a piano quartet. Thus a simplex is different to a clique. Sets of simplices have multidimensional connectivity, e.g. two high dimensional simplices can be connected through a triangular face, and simplices can be connected through a chain of intermediate triangle-connected simplices [2].

\subsection{Hypernetnetworks}

As with networks defined by binary relations, complex social systems require many higher relations to be taken into account in order to make sense of them. This can be done through the notion of hypersimplex in which the particular relation is explicit in the notation, e.g. $\left\langle a, b, c, d ; R_{\text {playing_bridge }}\right\rangle$ and $\left\langle a, b, c, d ; R_{\text {debating }}\right\rangle$. These hypersmplices have the same vertices but different relations. Any collection of hypersimplices is called a hypernetwork. Hypersimplices can represent wholes formed from their vertices as parts, and hypernetworks are an essential first step towards a way of representing the dynamics of multilevel systems [19].

\section{Networks and Policy}

Research over recent years has shown that much human behaviour at the microlevel takes place within and is constrained by networks. Furthermore analysis of social networks can provide information for policy in both the public and private sectors.

This is examined in some depth in Ormerod's book Positive Linking [28] which gives many examples in which people's economic and social decision making is determined by networks through influence and imitation. These include Asch's 1951 experiment [1] in which groups of eight subjects were asked to match the length of lines. The experiment was arranged with one subject and seven 'confederates'. The confederates answered first followed by the subject. In the first two rounds the subjects and the confederates all gave the correct answers. In the third round some of the confederates gave the same incorrect answer, but this time some of the subjects also gave the incorrect answer. Even though they were obviously wrong, three quarters of the subjects also gave the wrong answer at least once. This copying effect depends on the number of people giving the incorrect answer. "In one condition they put 16 naive persons in a room and had two confederates give wrong answers. The group responded with amusement at their errors. " [30].

In their book 'I'll have what she's having' Bentley et al. give an anthropological and social perspective on copying as an advantageous strategy [6]. They list some of the more well-studies strategies as: copy the majority; copy successful individuals; 
copy if better; copy good social learners; copy kin; copy friends; and copy older individuals. They illustrate this by saying that they themselves had copied this list from their colleague Kevin Laland, who himself had copied a list and added to it. And it is copied again here.

As Ormerod's chapter in this book makes clear, when it comes to making choices, copying is a much more convincing explanation for the decisions made than rational choice with perfect information [29]. This insight is of great potential value to policy makers because it gives new network-based methods of understanding the possible outcome of policy, and can help to shape policy. For example, many policies attempt to control behaviour by price applied to individuals (the vertices in networks) even when that behaviour is actually determined by social relationships (the links in networks). Sections 5.1-5.4 give examples of this.

\subsection{The Use of Financial Services}

A 2004 study by Meadows et al. is an early example of network ideas applied in policy [24]. In the context of Government proposals to eliminate financial exclusion, this research investigated the one in ten British adults not using mainstream financial services. Even though most of them were not in paid employment, many had bank accounts. It was found that non-consumers of financial services are distinguishable from consumers only by belonging to social networks where financial services usage is relatively low. The policy question was whether the financial institutions were reluctant to serve low-income customers, or whether the non-customers did not know that the services were available. By considering networks this study shed light on this question. "Use of financial services by the members of an individual's social network has a strong influence on their behaviour. Non-users are disproportionately drawn from social networks where few or no members have bank or building society accounts. This suggests that conventional marketing methods are not very successful in delivering information about financial services to non-users, and that there is an important information failure in these groups in the population" [24].

\subsection{Obesity}

In England $62 \%$ of adults and $28 \%$ of children aged between 2 and 15 are overweight or obese. Health problems associated with being overweight or obese cost the National Health Service more than $£ 5$ billion every year. The UK government's policy is that by 2020 there will be a downward trend in the level of excess weight in adults; and a sustained downward trend in the level of excess weight in children. [12] To achieve this it proposes to: encourage and help people to eat and drink more healthily, and to be more active. Its policy also includes giving advice on a healthy 
diet and physical activity, improving labelling on food and drink, and including calorie information on menus.

These policies address individuals as isolated vertices, but being overweight is a network phenomenon. The ego network of an obese person is likely to include more obese people than the ego network of a thin person. Despite the external social pressure against being overweight, cliques of people can be mutually forgiving when ordering another slice of cake, sharing a packet of biscuits, or eating chocolates.

In contrast to the government's approach, the private sector has developed a very effective method of weight control through the Weight Watchers organisation. Individuals wanting to lose weight can join Weight Watchers and immediately become part of a social network focussed on weight control. This involves imparting information not to the individual but to the group. Losing weight is a group objective, and individuals are encouraged by the success of others, formalised by ringing a bell when someone has achieved a milestone in their journey to achieve their desired weight. Instead of an isolated individual receiving impersonal advice, Weight Watchers have deliberately created mutually supportive locally networked communities in which the individual gains strength from the network.

\subsection{Smoking}

For more than a century it has been known that smoking cigarettes is a serious health hazard. Nonetheless, generation after generation have become smokers. In the postwar nineteen fifties and sixties smoking became fashionable, and the habit cascaded throughout populations. Half a century later, after decades of misinformation, many individuals addicted to smoking are trying to quit.

Christakis et al. write "The prevalence of smoking has decreased substantially in the U.S. over the past 30 years. ... Network phenomena appear relevant to smoking cessation. Smoking behavior spreads across close and distant social ties; groups of inter-connected people quit in concert; and smokers are increasingly marginalized socially. These findings have implications for clinical and public health interventions to reduce and prevent smoking" [10]. So, following network pressure in earlier decades to start smoking, today cascades of networked people are quitting smoking.

\subsection{Searching for Sadam}

When the American-led coalition invaded Iraq in 2003 one of the objectives was to capture Sadam Hussein and those who had played a major role in his regime. Towards this end the US Army produced a pack of playing cards to help troops identify the most-wanted targets, mostly high-ranking members of the Ba'ath Party and members of the Revolutionary Command Council. The cards reflected an 
approach based on political and administrative structures, but over time it became evident that those in this network structure did not know the whereabouts of Sadam Hussein. Another approach built a picture of the family and tribal networks and this led to the identification of an otherwise insignificant individual whose capture provided information on Saddam Hussein's whereabouts that led to his capture [32].

\section{Systems and Networks Theory Informing Policy}

Systems and network theory inform policy in complementary ways. Systems theory provides a way of modelling systems with many parts and many interactions and provides associated concepts, e.g. that feedback can be a reason for social systems having complicated behaviours that make them unpredictable. Systems theory is able to give powerful insights into possible futures that can usefully inform policy makers in their decision making.

In contrast networks provide structural insights into the behaviour of social systems. It is known that many social networks have long tailed degree distribution. This simple fact enables policy to be formulated in terms of identifying individuals with high degree since this may enable the formulation of more effective policies. For example, inoculating a high degree individual in a network of face-to-face meetings will be more effective at limiting contagion that inoculating people at random.

Another simple fact can guide policy makers, namely that some behaviour depends on individual vertex attributes while other behaviour depends on network attributes. For example, if I get a parking fine I may change my parking behaviour without consulting my social networks. In contrast, policies to discourage binge drinking may only be effective if they address whole networks.

In the era of big data, network motifs are useful in discovering structures and unearthing latent information. These are used extensively by companies like Google and Amazon to target people with information or advertisements. Generally motifs are structural relations on more than pairs of thing, i.e. they are hypersimplices in multilevel multiplex hypernetworks.

Systems and network theories are part of the wider science of complex systems science. In this book Dum and Johnson explain how this new science can work in the service of policy through Global Systems Science [13]. GSS adds to Complex Systems Science by providing a framework for scientists to work with policymakers. In particular it identifies policy informatics as a major means to connect science with policy, where this includes computer-based simulation, data, visualisation, and interactive interfaces. Importantly, GSS includes citizens in the science-based policy making process. 


\section{Conclusions: Systems, Networks, and Policy}

This chapter has introduced some basic ideas from systems theory. A major insight for policy makers is that social systems have feedback loops and that, even if they are all known, the interactions between them can be very complicated and difficult to comprehend without building a computational model. The dynamics are sensitive to small measurement errors, precluding precise predictions. Sometimes system diagrams give sufficient insight for policy purpose, but modelling and computer simulation are required to explore the possible future behaviours of complex multilevel social systems.

Modern network theory has given deep insights into the dynamics of systems, both in terms of the formation and loss of relational links, and the changing patterns of numbers over the vertices and edges. In particular it has been shown that the behaviour of individual human beings is highly dependent on their social networks. Network science adds to systems theory through its connectivity-based theory and structures.

Systems theory and network theory are fundamental tools for policy makers. Without them the outcome of policy is guesswork. Policy needs complex systems science but the science alone is not sufficient. To be effective, complex systems science has to provide policy makers and citizens with usable computer tools and data through policy informatics as developed in Global Systems Science.

Acknowledgements This work was supported by the Non-Equilibrium Social Science (NESS) Project funded by the Future and Emerging Technology (FET) unit of the European Commission, 2011-2014, and the UK Home Office through the Centre for Police Research and Learning at the Open University.

Open Access This chapter is distributed under the terms of the Creative Commons Attribution 4.0 International License (http://creativecommons.org/licenses/by/4.0/), which permits use, duplication, adaptation, distribution and reproduction in any medium or format, as long as you give appropriate credit to the original author(s) and the source, provide a link to the Creative Commons license and indicate if changes were made.

The images or other third party material in this chapter are included in the work's Creative Commons license, unless indicated otherwise in the credit line; if such material is not included in the work's Creative Commons license and the respective action is not permitted by statutory regulation, users will need to obtain permission from the license holder to duplicate, adapt or reproduce the material.

\section{References}

1. Asch, S.E.: Effects of group pressure on the modification and distortion of judgments. In: Guetzkow, H. (ed.) Groups, Leadership and Men, pp. 177-190. Carnegie Press, Pittsburgh (1951)

2. Atkin, R.H.: Multidimensional Man. Penguin Books, Hardmondsworth (1977) 
3. Ball, P.: The physical modelling of human social systems. ComPlexUs 2003(1), 190-206 (2003). doi:10.1159/000082449. http://philipball.co.uk/images/stories/docs/pdf/Complexus_ article.pdf

4. Barabasi, A.-L.: Linked. Plume, New York (2003)

5. BCF Taskforce: Better Care Fund: Policy Framework, Department of Health and Department for Communities and Local Government (2014) Published to gov.uk, in PDF format only. https://www.gov.uk/government/uploads/system/uploads/attachment_data/ file/381848/BCF.pdf

6. Bentley, A., Earls, M., O'Brien, M.: I'll Have What She's Having: Mapping Social Behaviour. MIT Press, Cambridge, MA (2011)

7. Berge, C.: Hypergraphs. Elsevier Science Publishers B.V., Amsterdam (1989)

8. Bignell, V., Fortune, J.: Understanding Systems Failures. Manchester University Press, Manchester (1984)

9. Bourne, J.: Bovine TB: The scientific evidence a science base for a sustainable policy to control TB in Cattle. An epidemiological investigation into Bovine tuberculosis. Final Report of the Independent Scientific Group on Cattle TB (2007). Presented to the Secretary of State for Environment, Food and Rural Affairs, June $2007 \mathrm{http} / / /$ webarchive.nationalarchives.gov.uk/ 20090330154646/www.defra.gov.uk/animalh/tb/isg/pdf/final_report.pdf

10. Christakis, N., Fowler, J.: Connected. Harper Press, London (2010)

11. DEFRA: The government's policy on bovine TB and badger control in England, Department for Environment, Food \& Rural Affairs, UK. 14 December 2011 https://www.gov.uk/governm ent/publications/the-government-s-policy-on-bovine-tb-and-badger-control-in-england https:// www.gov.uk/government/uploads/system/uploads/attachment_data/file/69463/pb13691-bovin etb-policy-statement.pdf

12. Department of Health: 2010 to 2015 government policy: obesity and healthy eating. Updated 8 May 2015 https://www.gov.uk/government/publications/2010-to-2015-government-poli cy-obesity-and-healthy-eating/2010-to-2015-government-policy-obesity-and-healthy-eating

13. Dum, R., Johnson, J., Global systems science and policy. In: Johnson, J., Nowak, A., Ormerod, P., Rosewell, B., Zhang, Y.-C. (eds.) Non-Equilibrium Social Science and Policy Springer, Berlin (2016)

14. Forrester, J.W.: World Dynamics. Wright-Allen Press. Cambridge, MA (1971)

15. Her Majesty's Treasury: Spending Review and Autumn Statement 2015 (2015). ISBN 9781474125833. https://www.gov.uk/government/uploads/system/uploads/attachment_ data/file/479749/52229_Blue_Book_PU1865_Web_Accessible.pdf

16. House of Lords Hansard: Bovine Tuberculosis. 3.11 pm, 23 Oct 2012, vol. 740, Col 142 (2012). https://hansard.digiminster.com/Lords/2012-10-23/debates/12102351000397/ BovineTuberculosis

17. ITV: Department of Health admits the NHS will collapse if improvements not made to health and social care. ITV Report, broadcast 4 March 2014 at 2:27pm (2013). http://www.itv.com/news/central/2014-03-04/department-of-health-admits-nhs-willcollapse-if-improvements-not-made-to-health-and-social-care/

18. Johnson, J.: Hypernetworks for policy design in systems of systems of systems. In: 2nd International Conference on Complex Sciences: Theory and Applications, 5-7 Dec 2012, pp. 179-189, Santa Fe. Springer, Berlin (2012)

19. Johnson, J.H.: Hypernetworks in the Science of Complex Systems. Imperial College Press, London (2014). http://oro.open.ac.uk/39122/

20. Kouvaris, N.E., Hata, S., Díaz-Guilera, A.: Pattern formation in multiplex networks Nat. Sci. Rep. 5 (2015). Article number: 10840 doi:10.1038/srep10840 http://www.nature.com/articles/srep10840kouvaris

21. Marsh, B.: Bed blocking by elderly patients rises (2015). Mail Online, 27-12-15 http://www.dailymail.co.uk/health/article-78550/Bed-blocking-elderly-patients-rises.html\#ixz z3vVOVmyVU

22. Meadows, D.H., Meadows, D.L., Randers, J., Brehens, W.W.: The Limits to Growth. Universe Books, New York (1972) 
23. Medows, D.H.: Thinking in Systems. Chelsea Green Publishing, White River Junction (2008)

24. Meadows, P., Ormerod, P., Cook, W.: Social networks: their role in access to financial services in Britain. Natl. Inst. Econ. Rev. 189, 99-109 (2004). http://www.paulormerod.com/ wp-content/uploads/2012/06/social-networks-role-in-access-to-financial-services.pdf

25. Merrick, J.: NHS feels the strain as hospital bed-blocking by elderly patients hits record levels. The Independent Newspaper, Sunday 22 Mar (2015). http://www.independent.co.uk/ life-style/health-and-families/health-news/nhs-feels-the-strain-as-hospital-bed-blocking-by-eld erly-patients-hits-record-levels-10125422.html

26. Moynihan, J.: In using charts to grapple with complex situations we should support dynamic thinking and avoid the over-simplistic. Letter published in The Times Newspaper, 3rd May (2010)

27. Nowak, A., Kacprzyk-Murawska, M., Serwotka, E.: Social psychology and the narrative economy. In: Johnson, J., Nowak, A., Ormerod, P., Rosewell, B., Zhang, Y.-C. (eds.) NonEquilibrium Social Science and Policy. Springer, Berlin (2016)

28. Ormerod, P.: Positive Linking. Faber \& Faber, London (2012)

29. Ormerod, P.: Economics. In: Johnson, J., Nowak, A., Ormerod, P., Rosewell, B., Zhang, Y.-C. (eds.) Non-Equilibrium Social Science and Policy. Springer, Berlin (2016)

30. Rollag, K.: Summary of the famous Asch experiment (1998). http://faculty.babson.edu/krollag/ org_site/soc_psych/asch_conform.html

31. Simon, H.: The Sciences of the Artificial. MIT Press, Cambridge (1965)

32. Wilson, C.: Searching for Saddam: the social network that caught a dictator (2010) slate.com, 7:35 am, Feb 23, 2010. http://www.slate.com/articles/news_and_politics/searching for_saddam/2010/02/searching_for_saddam_4.html 